\title{
The influence of diesel generators on frequency stability for isolated grids with high wind power penetration
}

\author{
Georgia Papaioannou ${ }^{1}$, Ignacio Talavera, Jutta Hanson \\ ${ }^{1}$ Department of Electrical Power Supply with Integration of Renewable Energies \\ Technische Universität Darmstadt \\ Landgraf-Georg-Str. 4, 64283 Darmstadt (Germany) \\ Phone: 0049 61511675697, e-mail: georgia.papaioannou@e5.tu-darmstadt.de
}

\begin{abstract}
The power generation of isolated power systems is based on expensive oil-fired or diesel conventional power plants. The combination of wind/diesel hybrid power systems is one of the most common proposed and promising solution for stability problems. This paper deals with the definition of an optimal combination for diesel generators from a technical point of view. On the one hand the extreme case that the power infeed equals the power demand without power reserves is considered and on the other hand a high amount of wind power is taken into consideration. The main goal is to find out if in case of dynamic events which are related to frequency stability, the power system would collapse for the operating conditions of minimum load and high wind power penetration. The scope is both to ensure system stability for different combinations of conventional generation, as well as to verify grid code requirements for providing active power support from Renewable Energy Sources that is required for the power system of the island of Lesbos in Greece.
\end{abstract}

\section{Key words}

Power systems, Power system stability, Isolated power systems, Wind power, Fault-Ride-Through

\section{Introduction}

Isolated power systems face challenges regarding their stability margins. The efforts to ensure stability for these grids have used different methods and proposals in the past [1]. In most cases the interest focuses on a high penetration of wind power and the related control strategies or in some cases regards the possibility of power production coming from Renewable Energy Sources (RES) exclusively [2] [3].

The centralised power infeed based on diesel generators determines the permissible RES penetration for most of the cases. The motivation of operating the power stations at technical minimum, so as to achieve the minimisation of fuel costs but also the requirements for the power reserves constitute a complicated task, as far as the definition of the conventional diesel generators in operation is concerned. Therefore in case of isolated grids an extreme careful investigation of their stability margins has to be performed before integrating RES.
In order to analyse the dynamic behaviour of the grid (MV and HV) and here especially the power generation units of such an electrical power system, we have modelled the grid of the island of Lesbos in Greece using the PowerFactory simulation software developed from DIgSILENT, aiming to perform stability investigations that will demonstrate the key factors influencing stability.

\section{Isolated Power Systems}

The influence of conventional power generation from diesel machines on power system stability in case of high penetration of RES will be demonstrated by defining cases studies for a particular isolated grid. This power system has been selected as it is a representative example of isolated systems, as far as power generation as well as structure and complexity is concerned. The electrical power system of the island of Lesbos and the requirements that have to be fulfilled and are given by the Greek Regulatory Authority for Energy (RAE) with reference to the integration of RES are presented.

\section{A. Power system topology}

The modelling process of the isolated power system is presented in detail in [4]. As referred, the model consists of a MV-grid of $20 \mathrm{kV}$ and a HV-line of $66 \mathrm{kV}$. The power infeed is based on the Autonomous Power Station (APS) consisting of 17 diesel generators with a rated power of 91.5 MVA. Power consumption is based on the data provided by the Distribution System Operator (DSO) and corresponds to a real loading situation of December 2012, which was $18.3 \mathrm{MW}$ for minimum load situation including losses. The reason for selecting this month is that after comparing load conditions from January 2011 until December 2013 it has been established that minimum load conditions as well as high wind speeds appeared in December 2012. These conditions enable the simulation of the highest wind power penetration. Figure 1 is a simplification of the modelled power system to give an overview of the power system's structure and components. It consists of four 
central double busbars of $20 \mathrm{kV}$ and four central busbars of $66 \mathrm{kV}$. The $66-\mathrm{kV}$-line serves the distribution of the power from the Autonomous Power Station (APS) to the 20-kV-Central Substation (CS) and finally to the consumers.

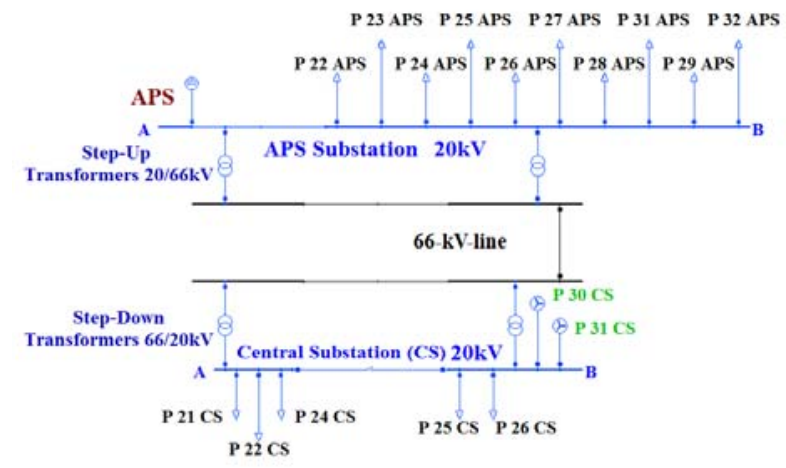

Fig. 1. Simplified diagram of Lesbos electrical power system

The 20-kV-APS Substation is modelled as a double busbar where both diesel generators and load feeders of the central city of the island are connected (P 26 APS, P 27 APS, P 31 APS, P 32 APS).

\section{B. Power system generation}

The power system supply is based on diesel generators of the APS and on Wind Power Plants (WPP). Information about both generation types is given below.

\section{1) Generators}

To enable a better understanding of the generators operation and to present the results in a structured way, we have introduced a concept of categorising the generators in three groups according to their rated power [4]. The group definition of the generators is based on the models and simplifies the process of analysing their dynamic behaviour. In Figure 2 the three defined groups are presented.

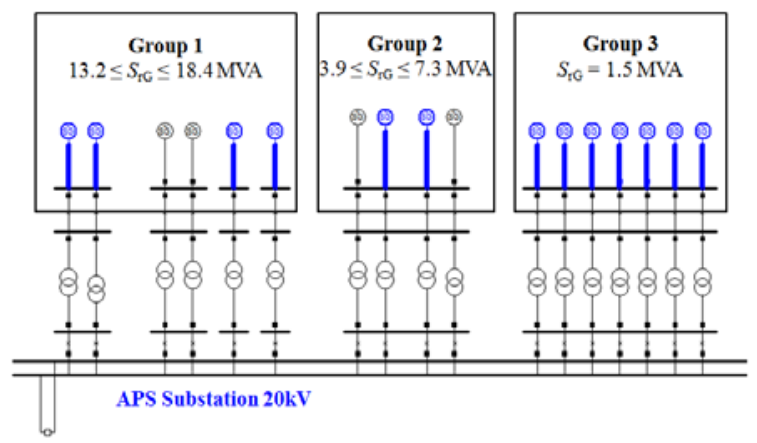

Fig. 2. Detailed structure of the generators of APS Substation

"Group 1" is composed of six generators (G03, G05, G06, G07, G08 and G09) with a rated power between 13.2 MVA and 18.4 MVA. Respectively "Group 2" (G01, G02, G04, and G10) consists of generators with a rated power between 3.9 MVA and 7.3 MVA, whereas portable generators of "Group 3" (G11 - G17) have a rated power of 1.5 MVA. The inertia time constant rated to $S_{\mathrm{rG}}$ is 4 seconds for all generators. The concept of grouping the generators enables the definition of different powerinfeed scenarios taking into consideration the operation of different diesel generators to demonstrate the influence of the choice for the generators in operation.

Voltage support and reactive power demand are served both from shunt capacitors installed at the feeders as well as at both central substations -APS and CS. The maximum amount of reactive power that can be provided from shunt capacitors installed at feeders is 8.1 Mvar. An amount of only 0.9 Mvar is located in APS feeders and the rest of the shunt capacitors are installed at the CS feeders, which aims to supply the reactive power at the loads located far away from the APS. Additionally directly at APS substation 12.9 Mvar and directly at CS 8.8 Mvar are installed. The connection or disconnection for the ones installed at the feeders depends on the loading condition of the power system.

\section{2) Wind Power}

The wind power infeed is located in four WPPs connected to the feeders according to the information provided in [4]. All of them consist of Enercon wind turbine generators; therefore the model of Enercon wind energy converter has been used. The dynamic behaviour of the model is described in detail in [5]. It is possible to achieve the most real modelling of RES by using the dynamic models of the wind generators that are already installed in the isolated power system.

Wind turbines are of a rated power of $0.6 \mathrm{MW}$ (E-40 Enercon Model) and 0.9 MW (E-44 Enercon Model). However the active power fed into the grid corresponds to the power coming from the power curve of the wind generators according to wind speed data provided from the Department of Geography of the University of Aegean.

\section{Grid code requirements for RES}

As far as the control of the wind turbines for network faults is concerned, the grid code focuses on the LowVoltage-Ride-Through (LVRT) requirements. This is the most important requirement regarding wind farm operation that has been recently introduced in the Greek grid codes. It is vital for a stable and reliable operation of power supply networks, especially in regions with high penetration of wind power generation. Faults in the grid can cause large voltage dips across wide regions and some generation units without this capability can be disconnected as a consequence [6].

A main requirement of the grid code is that symmetric or non-symmetric voltage dips are not allowed to lead to the disconnection of the WPP, if the voltage remains above the bold green line in Figure 3. Figure 3 shows that each generating unit shall remain transient stable and connected to the system without tripping for a threephase short circuit or any unbalanced short circuit in the transmission system with a fault clearing - time of up to 150 milliseconds and for a remaining voltage above $15 \%$ of the voltage before the fault. Throughout the operating 
range of the generating unit, these types of faults must not result in instability or isolation from the network. Furthermore, Figure 3 shows that power units shall be capable of continuous operation down to $90 \%$ of the voltage before the fault at the Point of Common Coupling (PCC) [7].

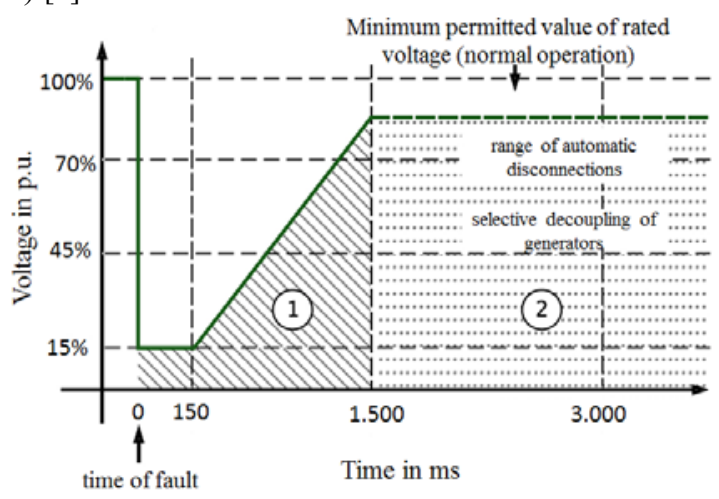

Fig. 3. Voltage requirements for LVRT in Greece [7]

\section{Load-Flow Scenarios}

In this section the considered steady-state simulation scenario and the power infeed assumptions are described and the corresponding results are presented. From the scenarios defined including RES, frequency stability is analysed for the scenario "Min. Diesel Generation - RES Min Load", which is one of the most crucial and therefore will be now taken into consideration [4]. Since the assumption of minimum diesel generation increases the possibility of a system collapse in case of a fault, three basic combinations for the generators are presented.

Generator G01 is defined as Slack node for all these cases to enable a comparison between the cases. Definition of these generator combinations is based on the efforts of maintaining system stability and exploring the optimal choice concerning generators in operation. Additionally this scenario of minimum load enables the simulation of the power system with power penetration corresponding to approximately $50 \%$. This is a very crucial scenario which has led to system collapses many times in the past. The first combination denoted as "Case 1" considers the operation of seven generators with the same rating belonging to "Group 3". The second combination, denoted as "Case 2", realises a mix between "Group 2" and "Group 3 " by considering the operation of half of the generators belonging to "Group 3" (G11, G12, G13) and G02 belonging to "Group 2". "Group 1" has not been considered, since only one generator could supply the demanded active power.

\section{A. Initial Conditions for the Dynamic Simulation}

As mentioned before we consider four cases for active power infeed. Table I presents the generators active power infeed cases as well as the allowed maximum active power infeed and the rated active power according to the data given by the Greek DSO [8]. The reactive power of the power system for minimum loading is completely supplied by the shunt capacitors and therefore the reactive power provided by the generators equals approximately zero and is not listed below.

Table I. - Infeed of generators: Active Power "Case 1" and "Case 2"

\begin{tabular}{|c|c|c|c|c|c|}
\hline & Generator & $\begin{array}{c}P_{\mathrm{G}} \text { in } \mathrm{MW} \\
\text { Case } 1\end{array}$ & $\begin{array}{c}P_{\mathrm{G}} \text { in } \mathrm{MW} \\
\text { Case } 2\end{array}$ & $\begin{array}{c}P_{\text {Gmax }} \text { in } \\
\text { MW }\end{array}$ & $\begin{array}{l}P_{\mathrm{rG}} \text { in } \\
\mathrm{MW}\end{array}$ \\
\hline & G01 & 3.8 & 3.5 & & \\
\hline (3) & G02 & $\begin{array}{c}\text { Out of } \\
\text { Operation }\end{array}$ & 3.5 & 4.5 & 5.9 \\
\hline & G11 & \multirow{7}{*}{0.8} & \multirow{3}{*}{0.8} & \multirow{7}{*}{1.1} & \multirow{7}{*}{1.3} \\
\hline & G12 & & & & \\
\hline$m$ & G13 & & & & \\
\hline $\overrightarrow{0}$ & G14 & & \multirow{4}{*}{$\begin{array}{c}\text { Out of } \\
\text { Operation }\end{array}$} & & \\
\hline$\dot{0}$ & G15 & & & & \\
\hline & G16 & & & & \\
\hline & G17 & & & & \\
\hline
\end{tabular}

For the normal operation the generators are allowed to feed the grid with an active power listed in the third column, but for a short-time they can reach the limits of the rated power. The operational limits are defined from the $P_{\mathrm{Gmax}}$ but in case of faults the generators would supply active power up to $P_{\mathrm{rG}}$.

Table II presents active power infeed from the WPPs for both cases respectively.

Table II. - Wind Power Plants: Active Power "Case 1" and "Case 2"

\begin{tabular}{|l|c|}
\hline \multicolumn{1}{|c|}{ Wind Power Plant } & $P_{\mathrm{WPP}}$ in MW \\
\hline WPP 1/ 2.7 MW & 1.4 \\
\hline WPP 2/ 2.7 MW & 1.4 \\
\hline WPP 3/ 4.8 MW & 3.2 \\
\hline WPP 4/ 4.2 MW & 2.8 \\
\hline
\end{tabular}

The power coming from the wind power plants corresponds to a wind speed of $10 \mathrm{~m} / \mathrm{s}$. Consequently, the active power coming from the conventional generation equals 9.4 MW and the one coming from wind power plants $8.8 \mathrm{MW}$ approaching the limit of $50 \%$ wind power penetration.

\section{Dynamic Simulations}

Dynamic simulations are organised according to the two proposed controlling methods regarding the governor models of the generators in operation. Basic concepts for diesel governors are the isochronous governor and the governors with speed droop characteristics. The isochronous governor ensures operation at constant speed and its main characteristic is to bring the frequency back to the nominal or scheduled frequency value after a dynamic event. For load sharing the speed droop governors have to be taken into consideration. Load sharing between two or more generators is realised for frequency changes according to their speed droop constant, which determines the change of power output for of frequency deviations. 


\section{A. Control methods}

Method A: All machines use the diesel governor DEGOV1 provided by the PowerFactory library, with a droop function and the same load sharing between all machines for "Case 1" and "Case 2".

Method B: The machines of "Group 2" will use the isochronous governor DEGOV without a droop function. All other generators use the DEGOV1 governor as described above. The reason why the generators of "Group 3" use the DEGOV1 is the fact that portable generators operate with a droop function in reality.

\section{B. Dynamic simulation scenarios}

Trip of active power as well as short circuit are simulated, as it is the common practice for stability studies in isolated grids. The electrical frequency is measured at APS busbar for all simulation events.

Investigetions Trip of active power:

1) Tripping of G11 represents a power loss of approximately $4.4 \%$ of total power. The simultaneous tripping of G11 and G12 represents a power loss of $8.8 \%$ and the one of G11, G12 and G13 simultaneously a power loss of $13.2 \%$.

2) A power loss of $15.4 \%$ from RES is initialised with a tripping of WPP 4. This will be the extreme case.

Short circuits:

1) A 3-phase short circuit at Bus A of the Central Substation (CS) is simulated. The duration is assumed to be $100 \mathrm{~ms}$.

\section{Dynamic Simulation Results}

Simulation results will be presented in this section with reference to the control method and according to the dynamic scenarios defined above.

\section{A. Method A: Diesel Governor Model with droop function}

In this section, all generators are equipped with a Woodward Diesel Governor Model with a droop function [4]. A droop constant of $5 \%$ has been used.

\section{1) Trip of active power}

The comparison of several generator trippings correspong to $4.4 \%, 8.8 \%$ and $13.2 \%$ power loss between "Case 1 " and "Case 2" is presented. The frequency response is presented in Figure 4 for "Case 1" and "Case 2".

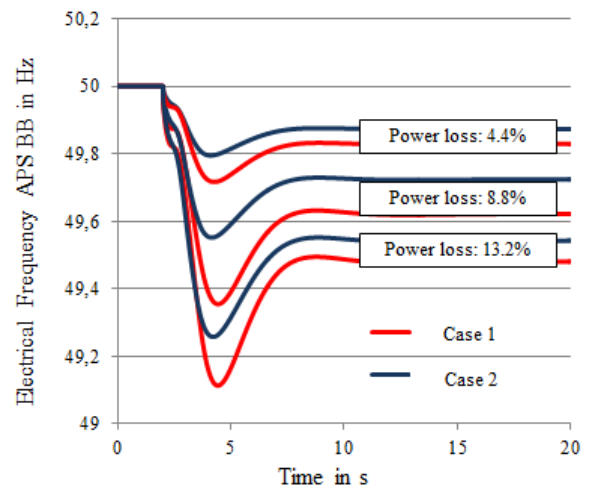

Fig. 4. Trip of $4.4 \%, 8.8 \%$ and $13.2 \%$ for "Case 1" and "Case 2" - Method A

The generator trippings does not influence frequency stability neither for $4.4 \%$ and $8.8 \%$ nor for $13.2 \%$ as the frequency overshoot is within the acceptable limits and depends only on active power loss [7]. As can be seen independent of the generators in operation the droop control Method A ensures frequency stability and leads to minor differences between the two cases.

The results of Figure 4 and 5 can be expected, since a power loss of $13.2 \%$ corresponds to $2.4 \mathrm{MW}$ whereas the remaining power that could be provided in short-term from the generators in operation is $2.8 \mathrm{MW}$ considering the limit of $P_{\mathrm{Gmax}}$ which can be violated for a short-time. Therefore the limit according to the rated power for both cases is $P_{\mathrm{rG}} 4.1 \mathrm{MW}$ (see Table I). Furthermore, since the inertia of the power system is the same for "Case 1 " and "Case 2" approximately the same and the droop constants are the same, the differences are small.

Consequently, a power loss of $2.8 \mathrm{MW}$ would push the power system's generators to their limits and therefore simulation results of the WPP 4 tripping are presented below in Figure 5.

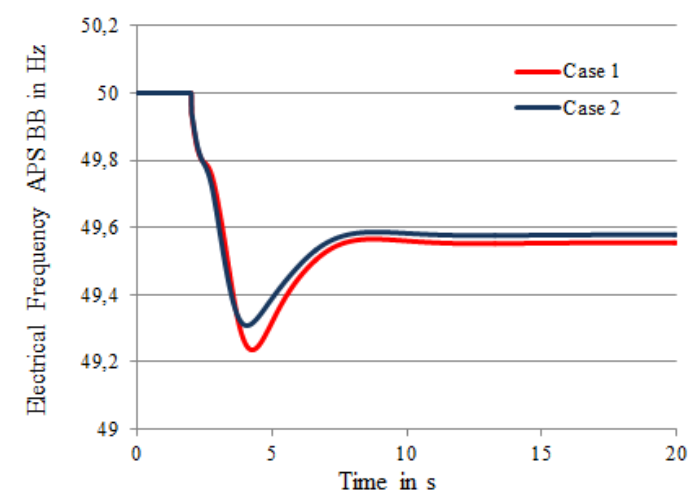

Fig. 5. Trip of WPP 4 for "Case 1" and "Case 2" - Method A

This WPP tripping does not influence frequency stability at all although active power loss is maximum that could be supported from the diesel generators for both cases. The fact that diesel generators are responsible for frequency control and continue operation result in the fact that frequency overshoot does not remarkably change in comparison to a $15.4 \%$ power loss coming from diesel generator trippings, although the active power lost is the maximum one that could be provided. 


\section{2) Short Circuits}

Until now has been shown that active power losses do not affect frequency stability independent of the combination with droop controlled generators. Although it could be stated that a control strategy based on droop functions seems to be very stable, simulation of short circuits has proven that this control method is not suitable for this isolated power system.

In case of short circuits this control strategy for the same load flow lead to a power system collapse independent of the combination of diesel engines that are in operation. None of the simulations converges using Method A. Instability occurs since the machines accelerate and none of them controls the frequency back to the nominal value and according to the droop a power balance cannot be reached for lower frequencies.

\section{B. Method B: Diesel Governor Model in isochronous mode}

The same dynamic simulation scenarios are simulated for the second control method.

\section{1) Trip of active power}

According to Method A, the frequency plot for the tripping of generators can be seen in Figure 6.

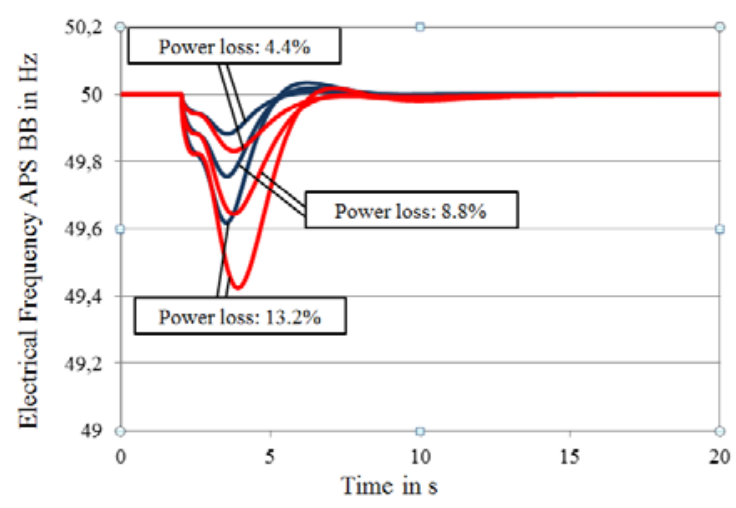

Fig. 6. Trip of $4.4 \%, 8.8 \%$ and $13.2 \%$ for "Case 1" and "Case 2" - Method B

The frequency overshoot has been remarkably reduced and in comparison to the former control Method A the frequency is supported more effectively. The second observation is that the frequency recovers to the nominal frequency without additional controlling. Additionally, the time required to reach a constant frequency after the generator trip is approximately six seconds, which is much longer using the former control Method A. This effect is caused by the nature of the isochronous governor, which stops adapting active power of the generator only after reaching the nominal frequency.

The frequency plot for tripping of WPP 4 can be seen in Figure 7.

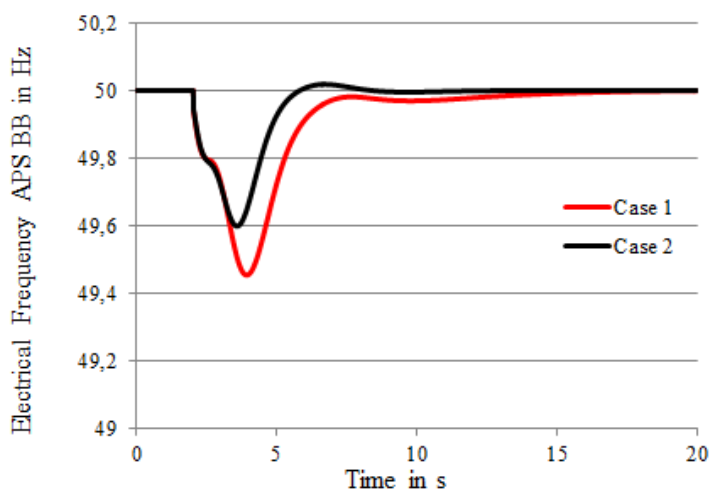

Fig. 7. Trip of WPP 4 for "Case 1" and "Case 2" - Method B

The same observations are also valid for tripping the WPP. The system remains stable and this control method succeeds to improve the frequency response.

\section{2) Short Circuits}

The dynamic scenarios concerning short circuits have proven the necessity of changing the control method for the diesel generators. For the initial conditions presented above, the power system needs a control method which prevents an immediate collapse once a short circuit happens. The frequency plot regarding a short circuit is presented in Figure 8.

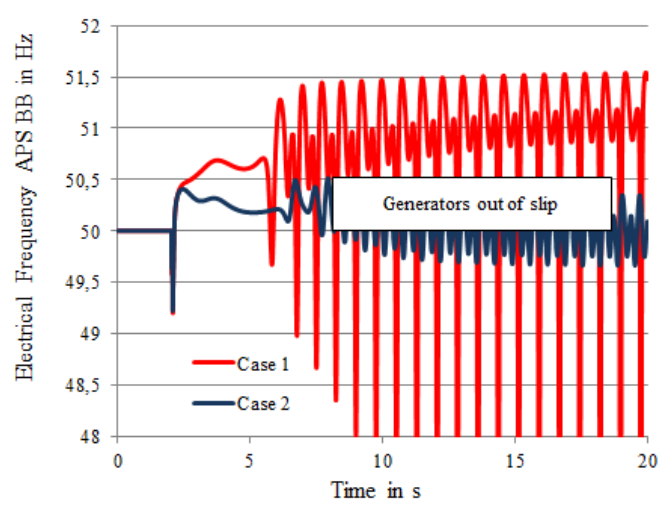

Fig.8 Electrical frequency after a short circuit for "Case 1" and "Case 2" - Method B

It can be seen that the electrical frequency goes up since the generators accelerate after the short circuit. Between "Case 1" and "Case 2" are small differences. The portable generators are all out of slip after five seconds of the time of fault for both cases. The reason is the fact that the WPP are disconnected once the short circuit happens and therefore the total active power demand cannot be provided only by the diesel generators. Their maximum rated active power is not sufficient to cover the half of the active power demand that has been supplied by the WPPs before the fault.

The disconnection of the WPPs is caused from the fact that the under voltage limit is violated according to the voltage requirements for LVRT in Greece, described in Figure 3. In Figure 9 is presented the voltage magnitude of the WPP 1 for "Case 1" and "Case 2" as a representative example. The same voltage plot is valid for all other three WPPs. 


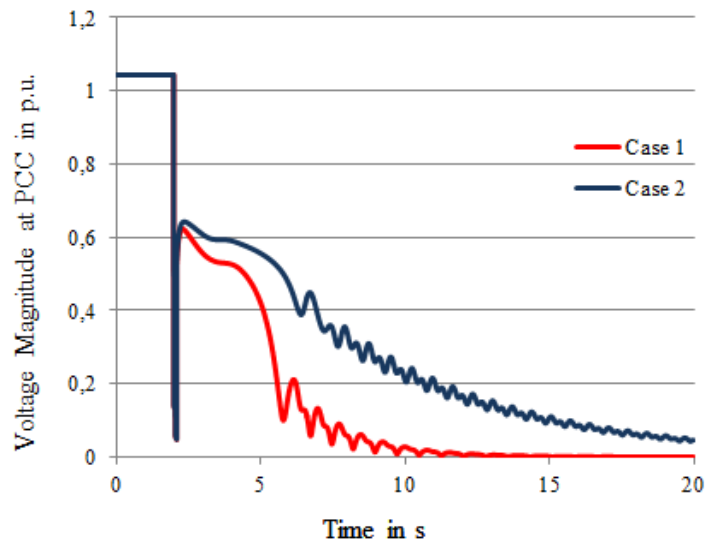

Fig.9 Voltage of WPP 1 at the PCC after a short circuit for "Case 1" and "Case 2" - Method B

It can be seen that the WPPs will be disconnected for both cases because the Enercon Models are so configured that the threshold for under-voltage clearance is set to be 0.8 p.u. and complies with the grid code requirements. Therefore, when the voltage is detected to be 0.8 p.u. the WPP will be automatically disconnected, leading to a total system collapse.

\section{Conclusion}

A high wind power penetration of about $50 \%$ in isolated grids is possible but there are crucial aspects that have to be considered for a stable operation. The most important are:

1. The classical approach of simulating only generator trippings in isolated power systems cannot ensure frequency stability for other faults, such as short circuits.

2. In isolated power systems the use of an isochronous governor is highly recommended and provides a more stable operation in comparison to a control strategy based exclusively on the droop characteristic.

3. The voltage requirements of the LVRT in case of such a high wind power penetration have to be further explored, since the WPPs do not remain connected after a 3-phase short circuit and do not supply active power leading to system collapse.

As a result, the Method A cannot ensure frequency stability for all dynamic simulation scenarios independent of the combination of diesel engines in operation and Method B cannot actually prevent a system collapse in case that the voltage requirements are not so adapted to prevent tripping of WPPs for this isolated power system. The combination of diesel generators of "Case 2" is generally better regarding the power system response, although cannot succeed in preventing instability.

To conclude, it has to be mentioned that the presented diesel generator's combinations offer a basis of discussion for the optimal operation of the power system and they cannot ensure an optimal economical solution for operation under these conditions.

\section{Further Works}

The following points that have to be further explored and are of interest:

1) Simulation of short circuits in other crucial places of the power system and with a longer duration.

2) Same generator tripping for other operation scenarios of diesel generation enabling a wind power penetration higher than $50 \%$.

3) Definition of an appropriate control method for maximum loading and for a higher wind power penetration as well.

4) Exploration of the appropriate voltage requirements for the LVRT for this isolated power system to prevent instability.

\section{Acknowledgement}

The authors acknowledge the Greek distribution system operator and especially the department of isolated grids for providing all data required in order to realise the model of the electrical power system of Lesbos, as well as their support during the modelling process and for the final validation. Furthermore we would like to acknowledge the company ENERCON for providing the WEC models for dynamic simulations. Last but not least we acknowledge Prof. Dr. Kostas Kalabokidis, Laboratory of Geography of Natural Disasters \& Forest Fires of the Department of Geography of the University of the Aegean, for providing the wind speed data of the island of Lesbos.

\section{References}

[1] I. D. Margaris, S. A. Papathanasiou, N. D. Hatziargyriou, A. D. Hansen and P. Sorensen, "Frequency Control in Autonomous Power Systems with High Wind Power Penetration”, in IEEE Trans. Sustainable Energy, Vol. 3, Issue 2 pp. 189-199, 2012.

[2] S A. Papathanasiou and N G. Boulaxis, "Power limitations and energy yield evaluation for wind farms operating in island systems", in Renewable Energy 31, pp. 457-479, Elsevier, 2006.

[3] R. Sebastiań and J. Quesada, "Distributed control system for frequency control in an isolated wind system", in Renewable Energy 31, pp. 285-305, Elsevier, 2006.

[4] G. Papaioannou, M. Fleckenstein, H. Zimmer and J. Hanson, "Dynamic Frequency Controlling for Isolated Island Power Systems", in ELECTRIMACS 2014, 19 -22 May 2014, Valencia, Spain

[5] G. Papaioannou, I. Talavera, H. Zimmer, J. Hanson, 'Influence of Fully Rated Converter Generator models on frequency stability in power systems with high wind power penetration', Poster Session, International Wind integration Workshop, 11-13 November 2014, Berlin, Germany

[6] C. Sourkounis, P. Tourou, "Grid Code Requirements for Wind Power Integration in Europe," Conference Papers in Energy, vol. 2013

[7] Regulatory Authority for Energy, (R.A.E.), "Code for operating non interconnected island electrical power systems", Athens, Greece, 2014

[8] Distribution System Operator (D.S.O.) Greece, Department of Island Grids, January 2013 